\title{
Clot in Transit in a Patient with COVID-19: Transesophageal Echocardiographic Guidance of Mechanical Cardiopulmonary Resuscitation
}

\author{
James Horowitz ${ }^{1}$, Eugene Yuriditsky ${ }^{1}$, Jan Bakker ${ }^{1}$, Gabriela Magda ${ }^{1}$, Felipe Teran ${ }^{2}$, and \\ Muhamed Saric ${ }^{1}$ \\ ${ }^{1} \mathrm{NYU}$ Langone Health \\ ${ }^{2}$ Hospital of the University of Pennsylvania
}

May 6, 2020

\begin{abstract}
The incidence of thrombotic complications among critically ill patients with COVID-19 is high despite thromboprophylaxis. We describe a case of a young, otherwise healthy patient admitted to our intensive care unit with COVID-19-associatred acute respiratory distress syndrome (ARDS). Despite an initial uncomplicated course, he suffered cardiopulmonary arrest with a right ventricular clot in transit (CIT) identified on point-of-care ultrasound (POCUS) and redemonstrated on transesophageal echocardiography (TEE). We highlight the association between COVID-19 and thrombosis as well as the utility of TEE during cardiac arrest for both diagnostic and therapeutic purposes.
\end{abstract}

\section{Case Presentation}

A 34-year-old man presented to our emergency department with a 1-week history of dyspnea and cough. On arrival, he was in acute respiratory distress with a respiratory rate of 40 breaths per minute with an arterial oxygen saturation of $88 \%$ when placed on a non-rebreather mask. Patient tested positive for severe acute respiratory syndrome-coronavirus 2 (SARS-COV-2) by polymerase chain reaction (PCR). Chest X-ray demonstrated diffuse bilateral patchy airspace opacities consistent with COVID-19 pneumonia. (Figure 1 ). He was emergently intubated, placed on mechanical ventilation, and admitted to the intensive care unit. Laboratory data on admission revealed markedly elevated inflammatory serum markers including a serum D-dimer of $438 \mathrm{ng} / \mathrm{mL}(<230 \mathrm{nl} / \mathrm{mL}$ upper limit of normal), a C-reactive protein of $188 \mathrm{mg} / \mathrm{L}$ (0$5 \mathrm{mg} / \mathrm{L}$ reference range), and a ferritin of $4,101 \mathrm{ng} / \mathrm{mL}(22-248 \mathrm{ng} / \mathrm{mL}$ reference range). Treatment with hydroxychloroquine, azithromycin, and tocilizumab was initiated and he was placed on subcutaneous heparin for thromboprophylaxis.

The patient remained on mechanical ventilation with a lung-protective ventilation strategy for the management of ARDS with a gradually improving course over the next 3 days. On the fourth hospital day, he developed supraventricular tachycardia and hypotension requiring emergent direct current cardioversion (DCCV). Point of care ultrasound (POCUS) revealed severe right ventricular (RV) dilation and hypokinesis with a clot in transit (CIT) in the right heart (Figure 2, Video 1 ).

Moments later, he developed pulseless electrical activity (PEA) and cardiopulmonary resuscitation (CPR) was initiated. A large CIT was redemonstrated on TEE in the right atrium during active CPR (Figure 3, Video 2 ). TEE was emergently performed to guide proper positioning of a Lucas mechanical CPR device 
(Jolife AB, Lund, Sweden). On TEE, the area of maximal compression (AMC) by mechanical CPR was compressing the left ventricular outflow tract (LVOT; Figure 4, Video 3 ). Under TEE guidance, the mechanical CPR device was moved caudally transitioning the AMS toward the body of the left ventricle (LV; Figure 5 , Video 4 ). Despite extensive resuscitative efforts including intravenous administration of tissue plasminogen activator (tPA), the patient developed an agonal rhythm (Figure 6, Video 5 ) and expired shortly thereafter.

\section{Discussion}

The incidence of thrombotic complications among patients with COVID-19 is high. In a study of 184 ICU patients with COVID-19 pneumonia, 31\% had thrombotic events despite prophylactic dose anticoagulation with pulmonary embolism (PE) being the most frequent (81\% of thrombotic events).11Klok FA, Kruip MJHA, van der Meer NJM, et al. Incidence of thrombotic complications in critically ill ICU patients with COVID-19. Thrombosis Research . 2020. https://doi.org/10.1016/j.thromres.2020.04.013 Patients with COVID-19 pneumonia have been found to have PE in the absence of predisposing risk factors.22Danzi GB, Loffi M, Galeazzi G, at al. Acute pulmonary embolism and COVID-19 pneumonia: a random association? Eur Heart J . 2020; https://doi.org/10.1093/eurheartj/ehaa254'33Xie Y, Wang X, Yang P, Zhang S. COVID19 complicated by acute pulmonary embolism. Radiology: Cardiothoracic Imaging. 2020; 2(2):e200067. We described a case of a previously healthy, young individual critically ill patient with COVID-19 ARDS who developed a large CIT and suffered cardiopulmonary arrest likely because of a massive PE.

Right ventricular thrombi, or clots in transit (CIT), are uncommon and occurring in $4 \%$ of unselected patients with $\mathrm{PE}$ but are associated with a considerable 27-45\% mortality rate.44Lai E, Alishetti S, Wong JM, et al. Right ventricular thrombus in transit: Raising the stakes in the management of pulmonary embolism. CASE: Cardiovascular Imaging Case Reports . 2019;3(6):272-276. 55Rose PS, Pubjabi NM, Pearse DB. Treatment of right heart thromboemboli. Chest . 2002;121(3):806-814.'66Konstantinides SV, Myer G, Becattini C, et al. 2019 ESC Guidelines for the diagnosis and management of acute pulmonary embolism developed in collaboration with the European Respiratory Society (ERS): The Task Force for the diagnosis and management of acute pulmonary embolism of the European Society of Cardiology (ESC). Eur Heart $J$. 2020;41(4):543-603. Optimal therapy for CIT is not defined as most data are based on case-series or registry results. However, meta-analysis data suggest superior results with thrombolytic therapy in these patients to alternative treatment modalities. ${ }^{5}$ 77Ferrari E, Benhamou M, Berthier F, et al. Mobile thrombi of the right heart in pulmonary embolism: delayed disappearance after thrombolytic treatment. Chest . 2005;127(3):1051-1053. As our patient was in cardiopulmonary arrest likely because of massive PE, tPA was the indicated therapy.

TEE has been used as a modality to evaluate patients during resuscitation; imaging can effectively diagnose the cause of arrest, alter therapy, and guide chest compressions.88Teran F, Dean AJ, Centeno C, Panebianco NL, Zeidan AJ, Chan W, Abella BS. Evaluation of out-of-hospital cardiac arrest using tranesophageal echocardiography in the emergency department. Resuscitation . 2019;137:140-147.'99Parker BK, Salerno A, Euerle BD. The Use of Transesophageal Echocardiography During Cardiac Arrests Resuscitation: A Literature Review. J Ultrasound Med.2019;38:1141-1151. In a study of 33 patients with out-of-hospital cardiac arrest presenting to the emergency department, $12 \%$ of those presumed to be in asystole were found to have fine ventricular fibrillation by TEE. Importantly, the AMC was identified to be over the aortic root or LVOT in $53 \%$ of cases. ${ }^{8}$ TEE imaging has demonstrated the left ventricular stroke volume was correlated with the location of the AMC. LV stroke volume improves when AMC is over the LV and away from the LVOT and the aorta.1010Hwang SO, Zhao PG, Choi HJ, Park KH, Cha KC, Park SM, et al. Compression of the Left Ventricular Outflow Tract During Cardiopulmonary Resuscitation.Acad Emerg Med . 2009;16:928933. In our case, the AMC of the mechanical CPR device was initially noted on TEE to be malpositioned over the LVOT. Under TEE guidance it was repositioned over the LV to improve chest compressions. 


\section{Conclusions}

We presented a case of a patient with COVID-19 ARDS who suffered cardiopulmonary arrest likely as a result of a massive PE and was found to have a CIT with severe RV dysfunction. We highlight the utility of point of care ultrasound as well as use of resuscitative TEE to establish the cause of cardiopulmonary arrest, as well as to guide CPR.

\section{References}

\section{Figure Legends}

Figure - Chest X-Ray

CXR demonstrates diffuse bilateral patchy airspace opacities consistent with COVID-19 pneumonia.

Figure - POCUS

Point of care ultrasound (POCUS) imaging in off-axis 4-chamber view demonstrates a severely dilated right ventricle (RV) and a large clot in transit in the right atrium (RA). LV, left ventricle. Video 1corresponds to this figure.

Figure - TEE

TEE in the approximate bicaval view demonstrates a large serpiginous clot in transit tumbling in the right atrium (RA) during different parts of the cardiac cycle during chest compressions. Video 2 corresponds to this figure.

Figure - TEE

TEE guidance of mechanical CPR. In this instance, the area of maximal compression (AMC) over the right ventricle (RV) is directed toward the left ventricular outflow tract (LVOT). This obliterates the LVOT and prevents proper left ventricular (LV) emptying. LA, left atrium.Video 3 corresponds to this figure.

Figure - TEE

TEE guidance of mechanical CPR. In this instance, the area of maximal compression (AMC) over the right ventricle (RV) is directed toward the body of the left ventricle (LV) and away from the left ventricular outflow tract (LVOT). This allows for proper LV emptying. LA, left atrium. Video 4 corresponds to this figure.

Figure - TEE

TEE demonstrates an agonal rhythm leading to cardiac standstill. RA, right atrium; RV, right ventricle; $\mathrm{TV}$, tricuspid valve. Video 5corresponds to this figure.

\section{Video Legends}

Video - POCUS

Point of care ultrasound (POCUS) imaging in off-axis 4-chamber view demonstrates a severely dilated right ventricle (RV) and a large clot in transit in the right atrium (RA). LV, left ventricle. Figure 2 corresponds to this video. 
Video - TEE

TEE in the approximate bicaval view demonstrates a large serpiginous clot in transit tumbling in the right atrium (RA) during different parts of the cardiac cycle during chest compressions. Figure 3 corresponds to this video.

Video - TEE

TEE guidance of mechanical CPR. In this instance, the area of maximal compression (AMC) over the right ventricle (RV) is directed toward the left ventricular outflow tract (LVOT). This obliterates the LVOT and prevents proper left ventricular (LV) emptying. LA, left atrium. Figure 4 corresponds to this video.

Video - TEE

TEE guidance of mechanical CPR. In this instance, the area of maximal compression (AMC) over the right ventricle (RV) is directed toward the body of the left ventricle (LV) and away from the left ventricular outflow tract (LVOT). This allows for proper LV emptying. LA, left atrium. Figure $\mathbf{5}$ corresponds to this video.

Video - TEE

TEE demonstrates an agonal rhythm leading to cardiac standstill. RA, right atrium; RV, right ventricle; TV, tricuspid valve. Figure 6 corresponds to this video.

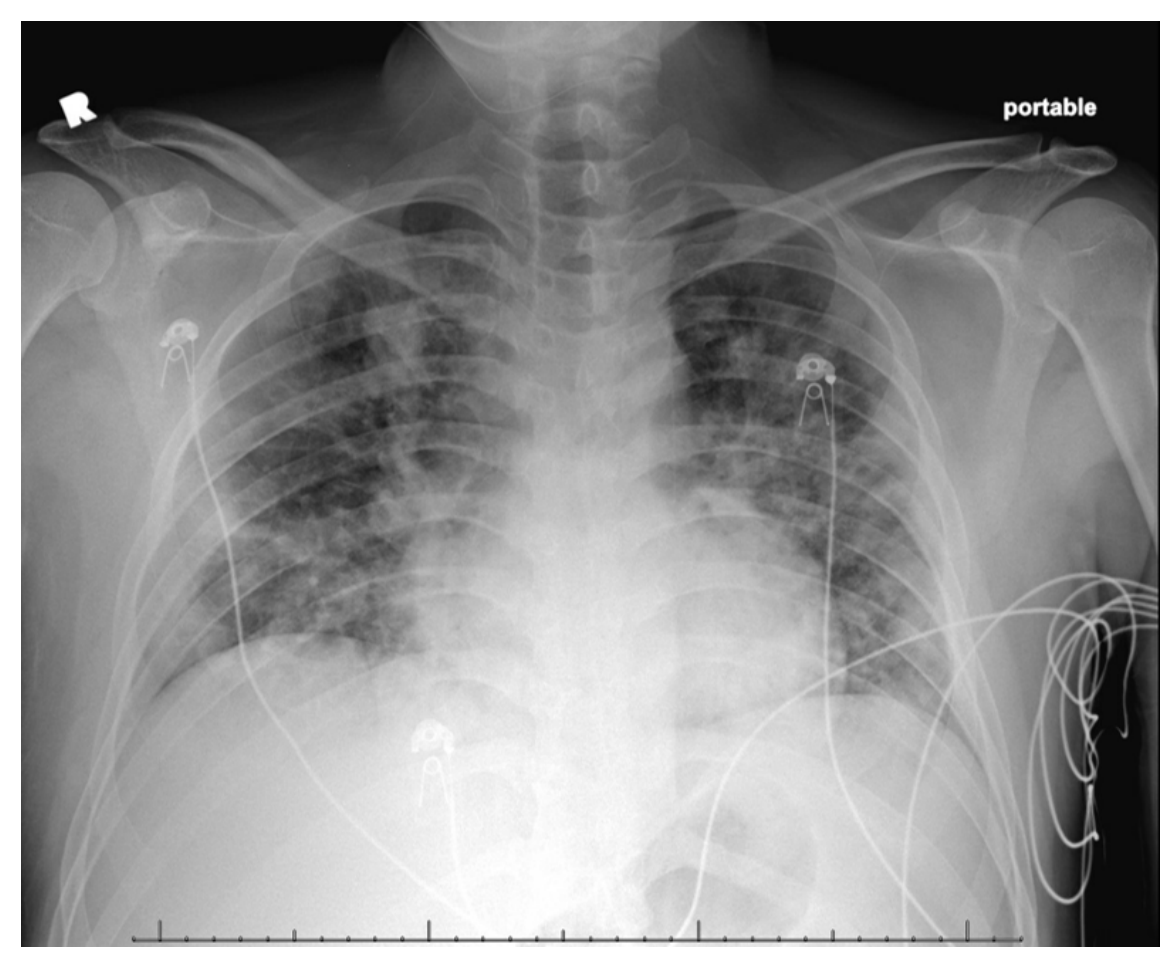




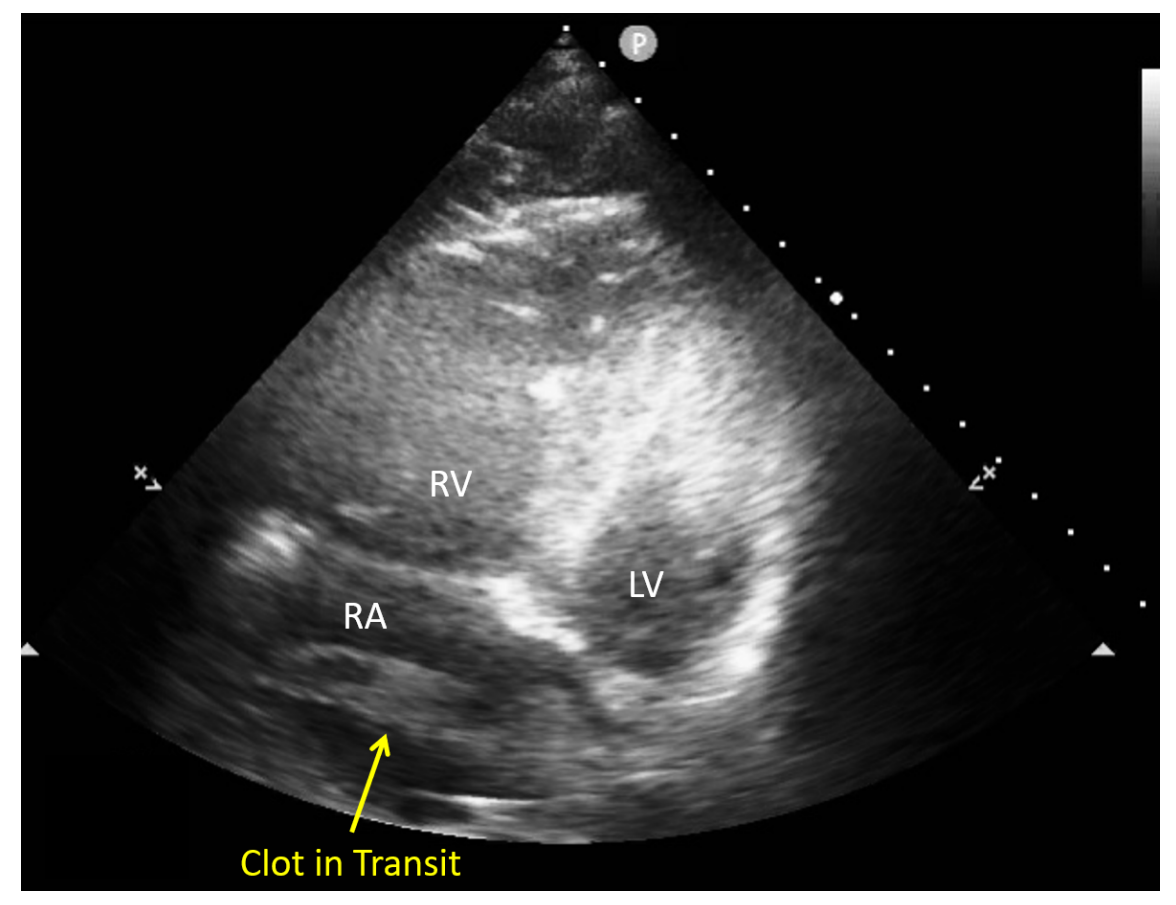



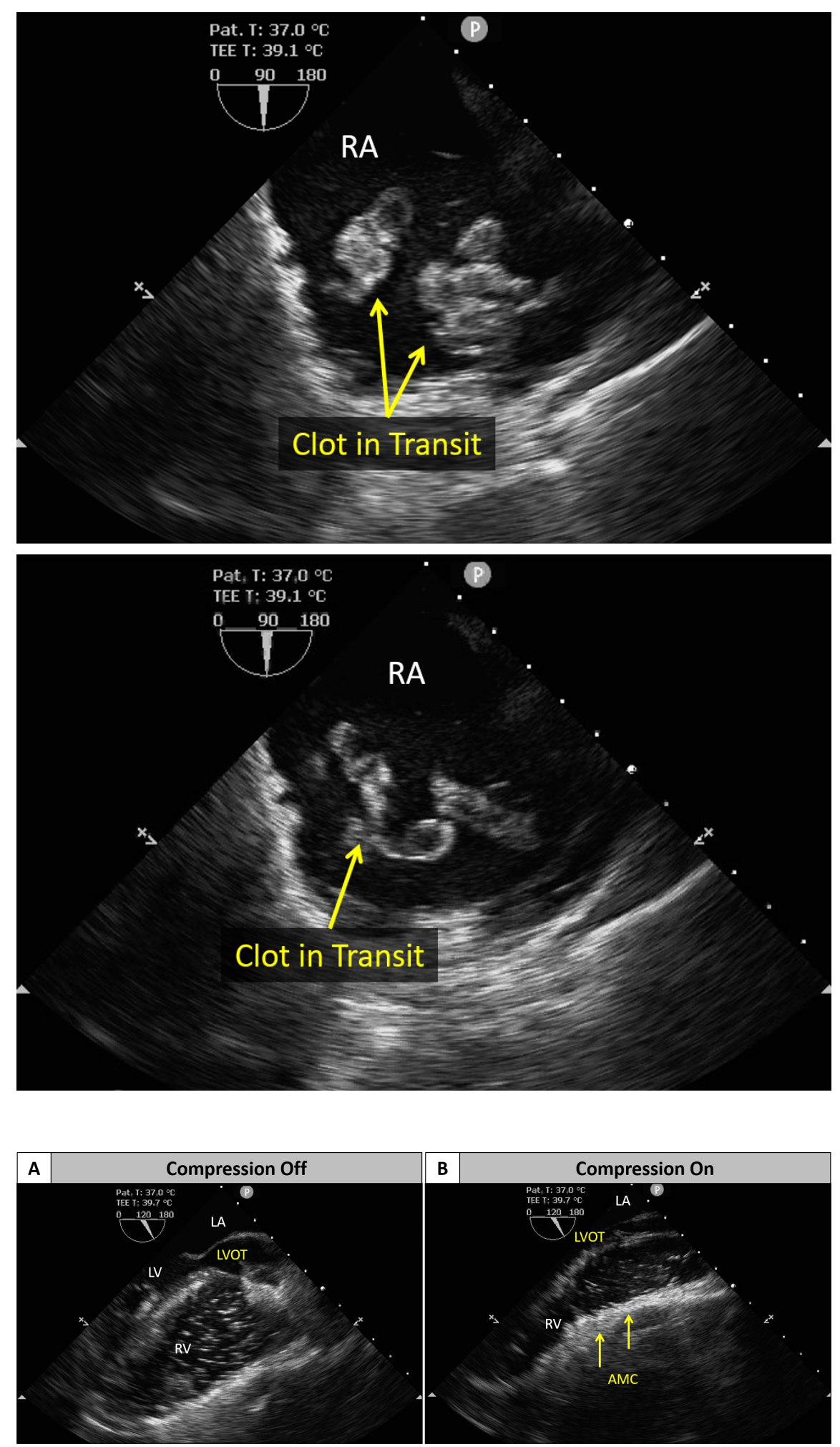

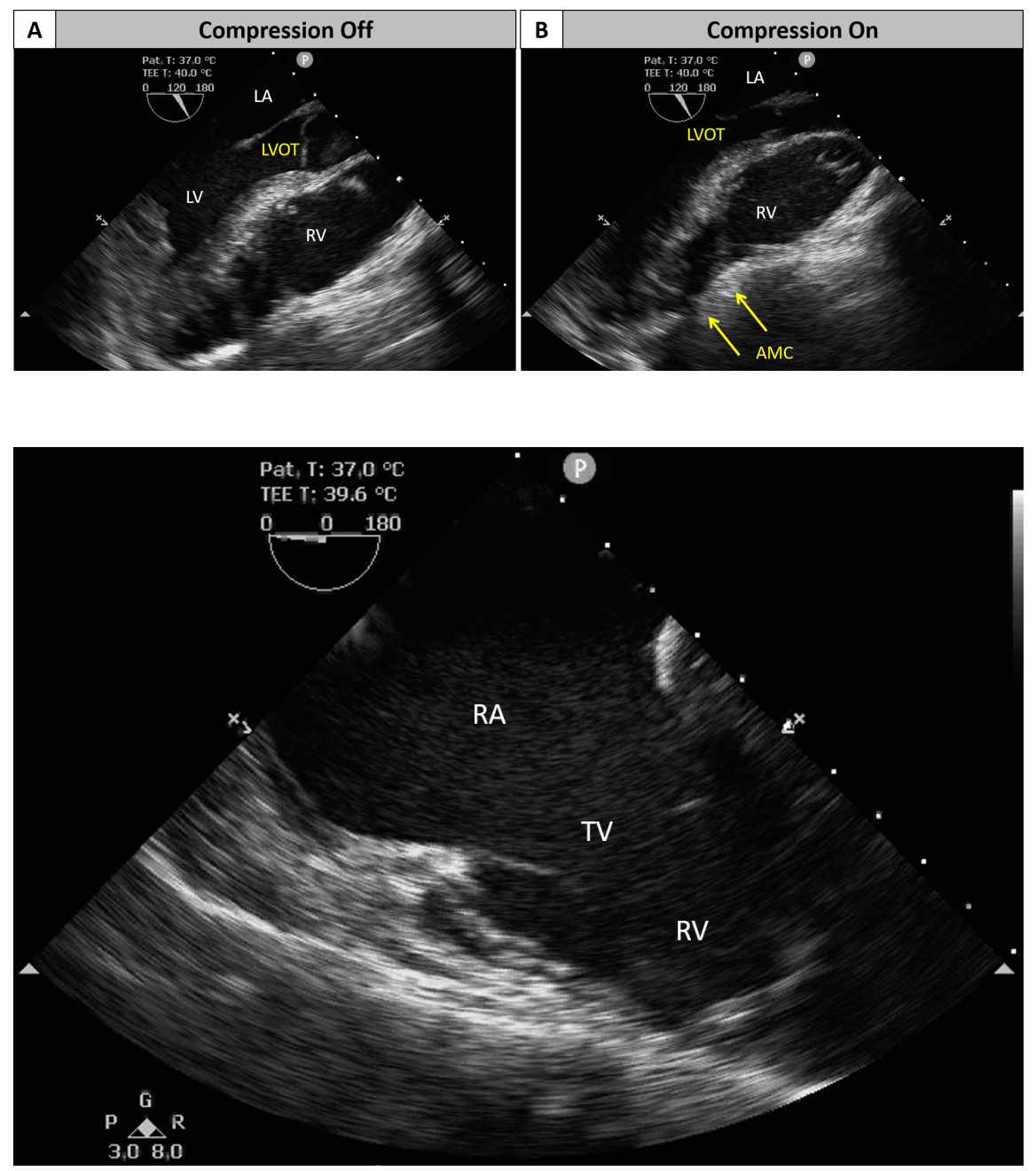